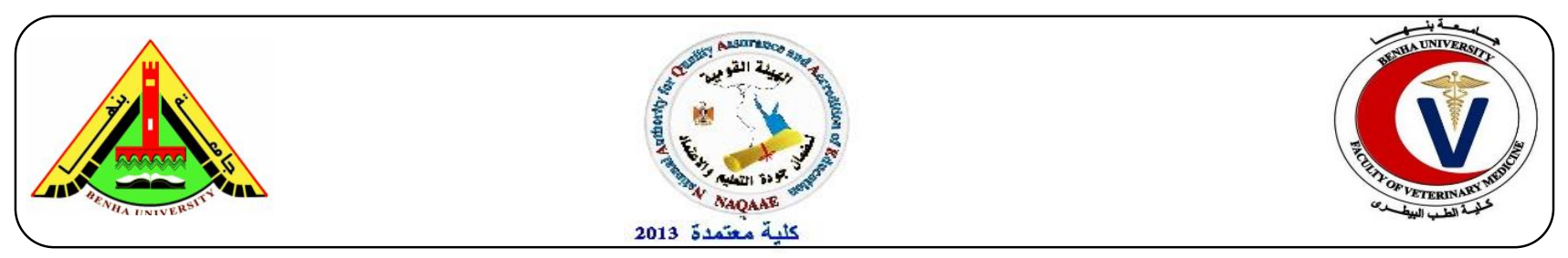

\title{
Field efficacy of concentrated inactivated bovine respiratory viral disease vaccine in cattle
}

\author{
El-Bagoury, G.F. ${ }^{1}$, Manal. A. Mansour ${ }^{2}$ and Iman. K.A. Kassem ${ }^{2}$ \\ ${ }^{1}$ Department of Virology, Faculty of Veterinary Medicine, Benha University \\ ${ }^{2}$ Veterinary serum and vaccine research institute, Abbassia, Cairo
}

\section{A B S T R A C T}

This work was carried out to evaluate the efficacy of locally prepared concentrated combined inactivated vaccines comprising BVD, BOHV-1, PI-3 and BRS viruses adjuvanted with aluminum hydroxide gel or montanide oil ISA206 under field condition.

The prepared vaccines adjuvanted aluminum hydroxide gel or montanide oil ISA206 were administered to 6-9-month-old seronegative calves through intramuscular (IM) route at different doses $2.5 \mathrm{ml}$ and $3 \mathrm{ml}$ respectively. The calves were boostered after two weeks and sera were collected two weeks after vaccination then monthly for $6^{\text {th }}$ month postvaccination. The induced antibodies were tested using Serum Neutralization Test (SNT). Our results revealed that, the concentrated vaccine has a significant effect on serum antibody levels by the $2^{\text {nd }} \mathrm{wpv}$ till $6 \mathrm{mpv}$. Moreover, the concentrated vaccine adjuvanted by montanide oil showed prolongation of antibody in comparison to the adjuvanted with aluminum hydroxide gel.

Keywords: Inactivated concentrated vaccine, Polyethylene Glycol, SNT.

(http://www.bvmj.bu.edu.eg) (BVMJ-36(1): 437-441, 2019)

\section{INTRODUCTION}

From an epidemiological point of view, it is difficult to incriminate one specific organism as a sufficient cause of most cases of respiratory disease. Rather, mixed infections involving together with one or more viruses, such as BoHV-1, P13, BRSV or Bovine Virus Diarrhea (BVD), are probably major components of the sufficient causes. Various management techniques and practices have been suggested to reduce the occurrence of respiratory disease, although few of these suggestions have been submitted to formal evaluation to determine their efficacy.

Therefore, multivalent vaccines are widely used to control the BRD. Live and inactivated vaccines with different combinations of antigens are available. The efficacy of these products is usually assessed by the measurement of the humoral and/or cellular immune response against one or more infectious agents under laboratory (Ellis et al., 1995; Fulton et al., 1995; Odendaal et al., 1997; Kerkhofs et al., 2004) or field conditions (West \& Ellis, 1997).

The use of inactivated vaccines against these respiratory diseases produces good results for protection of calves from Pneumo-enteritis and death (Knezevic et al., 1990). Inactivated virus 
vaccines have an advantage, because of safety issues. Also, concentration and purification of antigen in vaccine production has many advantages to produce large quantities of product that can be stored in smaller units, also improve immunological activity of viruses and decrease the dose given to the animal (Zeinab \& Nehal 2010)

This paper investigates the serological responses for a potent PEG concentrated inactivated combined vaccines containing BVDV, BOHV-1, PI3V, and BRSV adjuvanted with aluminum hydroxide gel and montanide oil ISA 206, vaccine under field condition.

\section{Materials and methods}

\subsection{Locally prepared PEG concentrated combine vaccine:}

Two batches of locally prepared PEG concentrated combine vaccine containing BVDV, BOHV-1V, PI-3V, and BRSV.one adjuvanted by montanide oil ISA 206 \& the other adjuvanted by aluminum hydroxide gel.

\subsection{Animals\& Experimental design (Field} application):

This experiment was conducted in two breeding farms (Beny Swif and El Salhya) in calves aged 6-9 month and about 150-200 kg body weight $(\mathrm{n}=50 / \mathrm{each}$ farm). The calves were divided into 2 groups (20 calves/group) in each farm. The calves received two successive doses of vaccines, two weeks apart intramuscularly $(2.5 \mathrm{ml}$ of gel vaccine, $3 \mathrm{ml}$ of oil vaccine) respectively. Also, another group (10 calves) in each farm inoculated with the same dose and route of physiological saline and act as control. All animals were periodically examined with monitoring their antibody titers.

\subsection{Sampling:}

Serum samples were collected from all calves of different tested groups just before $1^{\text {st }}$ vaccination, and then weekly post-vaccination until the first month and then monthly until the end of the experiment. All collected sera were inactivated at $56^{\circ} \mathrm{c}$ for $30 \mathrm{~min}$. in water bath for inactivation of non-specific antiviral substances then stored at $-20^{\circ} \mathrm{c}$ till used in serological test. All serum samples were serologically assayed for detection immune response and duration of immunity.

\subsection{Serum Neutralization Test (SNT):}

All serum samples were tested using serum neutralization test for detection of specific neutralizing antibodies against all vaccinal viruses of prepared gel\& oil adjuvants. SNT applied in micro-titration plate technique following the procedure described by Rossi and Kiessel, (1971). The Serum Neutralizing Antibody titers of the tested serum samples were expressed as $\log _{10}$ of the reciprocal serum dilution that protects $>50 \%$ of micro titration plate dilution wells, and these following the calculation formula of Reed and Muench, (1938).

\section{RESULTS}

Humeral immune response of vaccinated calves using SNT:

The mean neutralizing antibody in sera of calves vaccinated with concentrated inactivated combined gel\& oil adjuvante vaccine (Group 1\&2) were gradually increased from $14^{\text {th }}$ day post vaccination (DPV) within the minimal protective level for all viruses then reached highest level at $2^{\text {th }}$ month $\& 3^{\text {rd }} \mathrm{m}$ post vaccination (MPV) respectively, and still within the minimal acceptable titer of protective level till the end of the experiment (6 months) for all viral components of the prepared vaccine (BVD, BOHV-1, PI-3 and BRS virus). 
Field efficacy of concentrated inactivated bovine respiratory viral disease vaccine in cattle

Table1. Neutralizing antibody titer in calves vaccinated with concentrated (gel \& oil) inactivated combined vaccine in El-Salhya farm.

\begin{tabular}{|c|c|c|c|c|c|c|c|c|c|c|}
\hline \multirow{2}{*}{$\begin{array}{l}\text { Vaccine } \\
\text { applied }\end{array}$} & \multirow{2}{*}{ Dose } & \multirow{2}{*}{$\begin{array}{l}\text { Type of } \\
\text { Viruses }\end{array}$} & \multicolumn{8}{|c|}{ Mean serum neutralizing antibody tiers } \\
\hline & & & $0 d^{*}$ & $2 \mathrm{wpv} * *$ & $1 \mathrm{~m}$ & $2 \mathrm{~m}$ & $3 \mathrm{~m}$ & $4 \mathrm{~m}$ & $5 \mathrm{~m}$ & $6 \mathrm{~m}$ \\
\hline \multirow{4}{*}{$\begin{array}{l}\text { Aluminum } \\
\text { Hydroxide } \\
\text { gel }\end{array}$} & \multirow{4}{*}{$\mathrm{G} 1(2.5 \mathrm{ml})$} & BVD & 0.25 & 0.90 & 1.10 & 1.95 & 1.8 & 1.35 & 1.10 & 0.90 \\
\hline & & BOHV-1 & 0.4 & 1.00 & 1.25 & 1.8 & 1.9 & 1.4 & 1,25 & 0.60 \\
\hline & & PI-3 & 0.3 & 1,05 & 1.35 & 2.1 & 1.9 & 1.40 & 1.05 & 0.93 \\
\hline & & BRSV & 0.6 & 0.90 & 1.5 & 1.55 & 1.35 & 1.20 & 0.95 & 0.62 \\
\hline \multirow{4}{*}{$\begin{array}{c}\text { Montanide } \\
\text { oil } 206\end{array}$} & \multirow{4}{*}{$\mathrm{G} 2(3 \mathrm{ml})$} & BVD & 0.3 & 1.2 & 1.9 & 2.3 & 2.35 & 2.1 & 1.9 & 1.7 \\
\hline & & BOHV-1 & 0.45 & 0.9 & 1.5 & 2.1 & 2.4 & 1.95 & 1.8 & 1.5 \\
\hline & & PI-3 & 0.3 & 0.9 & 1.2 & 1.8 & 2.1 & 2.2 & 1.95 & 1.35 \\
\hline & & BRSV & 0.0 & 0.6 & 1.22 & 1.65 & 1.95 & 2.1 & 1.80 & 1.24 \\
\hline
\end{tabular}

*1st dose, **2nd dose.

Minimum acceptable titer of protective level expressed in $\log _{10}$ against, BVDV=0.9, BOHV-1V $=0.6$, PIV-3=0.6, BRSV=0.6 (Fulton et al., 1995).

Table 2. Neutralizing antibody titer in calves vaccinated with concentrated (gel \&oil) inactivated combined vaccine in Benisuef.

\begin{tabular}{|c|c|c|c|c|c|c|c|c|c|c|}
\hline \multirow{2}{*}{$\begin{array}{l}\text { Vaccine } \\
\text { applied }\end{array}$} & \multirow{2}{*}{ Dose } & \multirow{2}{*}{$\begin{array}{l}\text { Type of } \\
\text { Viruses }\end{array}$} & \multicolumn{8}{|c|}{ Mean serum neutralizing antibody tiers } \\
\hline & & & $0 \mathrm{~d}^{*}$ & $2 \mathrm{wpv} * *$ & $1 \mathrm{~m}$ & $2 \mathrm{~m}$ & $3 \mathrm{~m}$ & $4 \mathrm{~m}$ & $5 \mathrm{~m}$ & $6 \mathrm{~m}$ \\
\hline \multirow{4}{*}{$\begin{array}{l}\text { Aluminum } \\
\text { Hydroxide } \\
\text { gel }\end{array}$} & \multirow{4}{*}{$\mathrm{G} 1(2.5 \mathrm{ml})$} & BVD & 0.6 & 0.6 & 1.2 & 1.55 & 1.35 & 1.22 & 0.92 & 0.90 \\
\hline & & BOHV-1 & 0.45 & 0.65 & 0.90 & 1.38 & 1.21 & 1.05 & 0.81 & 0.57 \\
\hline & & PI-3 & 0.3 & 0.9 & 1.25 & 1.8 & 1.5 & 1.38 & 1.05 & 0.62 \\
\hline & & BRSV & 0.24 & 1.05 & 1.2 & 1.57 & 1.45 & 1.20 & 0.75 & 0.55 \\
\hline \multirow{4}{*}{$\begin{array}{c}\text { Montanide } \\
\text { oil } 206\end{array}$} & \multirow{4}{*}{$\mathrm{G} 2(3 \mathrm{ml})$} & BVD & 0.33 & 0.91 & 1.20 & 1.81 & 2.10 & 2.21 & 1.93 & 1.80 \\
\hline & & BOHV-1 & 0.3 & 1.2 & 1.62 & 2.46 & 2.4 & 2.56 & 1.98 & 1.68 \\
\hline & & PI-3 & 0.45 & 1.08 & 1.74 & 2.40 & 2.40 & 2.22 & 1.38 & 1.25 \\
\hline & & BRSV & 0.40 & 0.9 & 1.7 & 2.34 & 2.1 & 1.80 & 1.62 & 1.44 \\
\hline
\end{tabular}

$* 1$ st dose, $* * 2$ nd dose.

Minimum acceptable titer of protective level expressed in $\log _{10}$ against, BVDV=0.9, BOHV-1V = 0.6, PI-3V=0.6, BRSV= 0.6 (Fulton et al., 1995).

\section{DISCUSSION}

The effectiveness of a vaccine to prevent disease depends on the vaccine being potent and on its proper administration to individuals capable of responding. Useful techniques are available to test the potency of vaccines and the response of the host. Potency testing is important in monitoring the production of vaccines (to maintain stipulated standards), and their transport through the "cold chain". In the latter instance, vaccines from the field are retrieved and tested to ensure that they have not lost their potency.
Successful vaccination will be reflected on protection from morbidity and mortality against specific field virus and build immune foundation in calves. On the other hand, vaccination failure against these viruses causes severe economic losses and could not diagnose easily as the main cause of this failure is complex and depends on different complicating factors.

The present study was carried out to evaluate the efficacy of concentrated combined inactivated vaccines comprising BVD, BOHV-1, PI-3 and BRS viruses adjuvanted 
with aluminum hydroxide gel and montanide oil ISA206 under field condition.

This trial gave satisfactory results concerning the laboratory evaluation of the prepared combined inactivated oil adjuvant for sterility, safety and potency testing. Sterility test of the prepared oil vaccine showed complete absence of any contamination on the inoculated media for 2 weeks post inoculation. Also, the results of safety test showed a good safety profile for both gel \& Montanide oil adjuvants even in calves inoculated with 10 times of vaccinal dose showed neither local nor systemic post vaccinal reaction and no development of any clinical signs or any abnormalities till the end of the experiment.

Regarding potency evaluation of the prepared concentrated inactivated gel \& oil adjuvant under field condition.

The titer of neutralizing antibodies which was detected in the sera of vaccinated calves is much increased in PEG concentrated vaccine adjuvanted by both gel and oil where vaccinated calves gave high immune response as the antigen payload in PEG concentrated vaccine enhanced the immune response. This result indicated high potency of the concentrated vaccine which is adequate to protect susceptible animals from infection.

Estimation of humeral immune response to the inactivated combined vaccine showed that the mean antibodies titers were gradually increased from 2 weeks post vaccination and reached to the highest level at $2 \mathrm{~m}$ in gel $\& 3 \mathrm{~m}$ in oil and still in a protective level till the end of the experiment for all viruses' components of the vaccine compared to the non-vaccinated group as measured by SNT. and the prepared combined inactivated vaccine adjuvanted with the Montanide oil (ISA206) has been proved greatly effective giving duration of immunity more than that of the concentrated adjuvanted with Aluminum Hydroxide gel which gives a duration of up to $6 \mathrm{MPV}$. This agreed with the studies which reported that the minimum accepted neutralizing antibody titers were 0.9 $\log _{10}$ for BVDV and $0.6 \log _{10}$ for BOHV-1V, PI-3V, and BRSV (Fulton et al, 1995). Duration of immunity elicited by aluminum hydroxide gel vaccine was short-lived and antibody concentration rapidly falls over periods of 4-6 months after administration (Ellis et al, 2005, El-Bagoury et al, 2012)

The previous results revealed that the immune response against the components of the vaccine prepared from PEG concentrated viruses was greater than that prepared from unconcentrated virus, this result agreed with (Lyer et al 2001) who found that vaccines formulated with virus purified with $8 \%$ PEG were more immunogenic than the vaccines formulated with untreated harvest viruses.

\section{Conclusion}

In conclusion, concentration of viruses by PEG improves the quality of antigen used in the vaccine formulation to produce a good quality combined inactivated vaccine that was able to induce detectable levels of specific antibodies against BVDV, BOHV-1V, PI-3Vand BRSV and remained for long duration more than 6 months \& also, oil adjuvanted vaccine was more immunogenic than the gel adjuvanted one.

\section{REFERENCES}

El-Bagoury, G.F., El-Nahas, E.M., AbdElfadiel, M. R., Ghaley, H. M. 2012. Evaluation of maternal antibody in calves borne from cow dam vaccinated with inactivated pnumo-5 vaccine. BVMJ, 23 (2): 9-15.

Ellis, J. A., Hassard, L. E. and Morley, P. S. 1995. Bovine respiratory syncytial virus-specific immune responses in calves after inoculation with commercially available vaccines. J. Am.Vet. Med. Assoc. 206, 354-361.

Ellis, j., West, K.H., Waldner, C., Rhodes, C. 2005. Efficacy of saponin-adjuvanted 
inactivated respiratory syncytial virus vaccine in calves. Cand. Vet. J. 46: 2, 155-162.

Fulton R. W., Confer, A. W., Burge L. J., Perino L. J., d'Offay J. M., Payton M. E. and Mock R. E. 1995. Antibodies responses by cattle after vaccination with commercial viral vaccines containing BHV-1, BVDV, PI3, BRSV immunogens and subsequent revaccination at day 140 . Vaccine, 13: 725-733.

Kerkhofs, P., Tignon, M., Petry, H., Mawhinney, I. and Sustronck, B. 2004. Immune responses to bovine respiratory syncytial virus (BRSV) following use of an inactivated BRSV-PI3-Mannheimia haemolytica vaccine and a modified live BRSV-BVDV vaccine. Vet. J. 167, 208210.

Knezevic, N.; Kosanovic, P. and Rogan, D. 1990. Immunoprophylaxis of respiratory diseases of cattle with inactivated vaccine. III. Study of the immunogenicity of a bivalent inactivated oil vaccine against BOHV-1 and PI3. Veterinarski Glasnik, 44 (7): 503-512
Lyer A.V; Ghosh S; Singh S.N. and Deshmukh R.A. 2001. Evaluation of three ready to formulate oil adjuvants for FMD vaccine production. Vaccine 19 10971105

Odendaal, M. W., Morris, S., du Preez, E. and Aitchison, H. 1997. The humoral immune response in cattle after immunization with a multivalent BOHV-1/PI3/Pasteurella haemolytica A1leukotoxin vaccine. Onderstepoort J. Vet. Res. 64, 205-212.

Reed, L. J. and Muench, A. 1938. A simple method of estimating fifty percent and points. Amer. J. Hyg., 27: 443-445.

Rossi, C. R. and Kiessel, G. K. 1971. Microtitre tests for detecting antibody in bovine serum to PI3V, BOHV-1V and BVDV. Microbiol., 22: 32-36.

West, K. and Ellis, J. 1997. Functional analysis of antibody responses of feedlot cattle to bovine respiratory syncytial virus following vaccination with mixed vaccines. Can. J. Vet. Res. 61, 28-33

Zeinb, TS. Salama and Nehal S Abdelrahman. 2010. Preparation of inactivated purified concentration canine distemper vaccine. Zag. Vet. j. 40 (2): 95-99. 